\title{
A COMPARISON OF APPOINTMENT SCHEDULES IN A HOSPITAL RADIOLOGY DEPARTMENT
}

\author{
S. D. WALTER \\ Department of Epidemiology and Community Medicine, University of Ottawa, Canada
}

There has been a growing awareness in recent years of the need for health administrators to consider the objectives and welfare of both patients and staff when formulating working policy, and, in particular, the widespread introduction of appointment schemes in a wide range of clinics and general practice surgeries has been an important instrument in balancing the time wasted by doctors (in waiting for patients to arrive) and patients (in a queueing system). This paper considers some computer simulations of such appointment schemes and describes some aspects of the queueing systems that they represent, with particular reference to radiology departments.

\section{Data Collection and Model Construction}

In research involving stochastic systems of the type seen in clinic situations, it is often desirable to construct a model embodying as many as possible of the empirical characteristics observed. By using a simulation model, it is possible to include more empirical factors than in a simpler, but solvable theoretical model, but the more factors we include, the more we must concentrate on simulated systems with a close similarity to a practical example from which we may validate the model. There appears to be an antithesis between the precision and comprehensiveness of the model used, but this may be simply a reflection of the present state of knowledge, rather than something intrinsic in the models themselves.

Examples of simulation models of clinics are provided by Jeans, Berger, and Gill (1972), Fraser (1969), and Bailey (1952), while Pike (1963a) demonstrates a more theoretical approach to an outpatient queueing system. The model used here is described below, beginning with the pattern of patient arrivals and the structure of the service mechanism. The radiodiagnostic department in a modern hospital, being one of the larger departments and providing a specialized service, has a highly heterogeneous input of work derived from most of the other health service units in the area, namely, the hospital wards and clinics, other hospitals, doctors, and the public directly. The patients may be divided into three main groups: first, those who have appointments; secondly, those who do not have appointments but whose arrival patterns may be somewhat predictable from experience and knowledge of the working routine of the patient origin (e.g., another specialist clinic); finally, patients arriving as casualty cases in a random fashion. A more detailed discussion of these classifications is made in the Nuffield Provincial Hospitals Trust (1962) report.

A patient's service time, or the time needed to administer the treatment required, is well known by any practising medical worker to depend on many characteristics of the patient, for example, age, sex, illness classification, the general state of health, physical mobility, and so on. Nevertheless, it is often assumed for simplicity in both theoretical and simulation work that the service time follows a distribution which is the same for all patients. Bailey (1952), when considering appointment scheduling problems in outpatient and general practice clinics, used an Erlangian or gamma type distribution which may be fitted to a wide variety of empirical data, and others have also adopted this distribution, e.g., Pike (1963b) and Fraser (1969).

In this study, a gamma distribution was found to describe adequately the overall distribution of chest radiographic examination times but the distributions for particular classes of patient were significantly different. The chest radiograph was chosen for special consideration as it is an examination commonly carried out on patients of all types, and is fairly brief, making it possible to collect data quite rapidly. At the Royal Infirmary, Edinburgh, where the work was carried out, one room was set aside almost exclusively for chest radiographs and a flow of patients was present almost always. In the course of a full working week, some 256 examinations were observed during a random sample of busy and slack periods at various hours of the day. Some 33 of these used a miniature machine when only a minor investigation or routine checkup was required. The examination time 
recorded for each patient was the time spent in the $x$-ray room; in Edinburgh, patients waited in an area immediately adjacent to this room, and so the flow of patients in and out was effectively a continuous process at times when a queue of patients was present. Chest work at the Royal Infirmary provided an estimated $39 \%$ of the total work load of approximately 120,000 examinations annually (Source: R.I.E. Diagnostic Radiology Department Annual Returns). The independent parameters characterizing the gamma distribution are, in this case, the mean examination time, and a quantity $k$ indicative of the general 'shape' of the distribution, a higher value of $k$ indicating a less variable distribution. When the data on the service times of the 223 patients examined on the ordinary $x$-ray machine were considered, the maximum likelihood estimates of the mean and $k$ were evaluated as 2.72 minutes and 2.82 respectively. The observed distribution of examination times and the expected gamma distribution with these parameter values are shown in Table I; a goodness-of-fit test gave $\chi^{2}{ }_{10}=15 \cdot 2$ $(P=0 \cdot 1)$.

When the observations are grouped by the patients' ages, some expected patterns emerge, as in Table II, older patients requiring on average considerably more staff time for the examination than younger ones. A substantial proportion of the younger age group patients were examined on the miniature film machine; these patients tend to be fit, and thus mobile, more responsive to instruction by staff, and hence tend to require less time for the examination. When the data from all patients are pooled, the tendency for examination time to increase with age becomes much more marked. Also, the variances of the times for patients aged 60 or more and under 20 were both significantly higher $(P<0.01$ and $P<0.05$ respectively) than

TABLE I

OBSERVED AND THEORETICAL DISTRIBUTIONS OF TIME TO PERFORM CHEST RADIOGRAPH

\begin{tabular}{|c|c|c|c|}
\hline $\begin{array}{c}\text { Time Interval } \\
\text { (minutes) }\end{array}$ & & $\begin{array}{c}\text { Number of Patients } \\
\text { in Sample }\end{array}$ & $\begin{array}{l}\text { Expected Number } \\
\text { with Gamma } \\
\text { Distribution }\end{array}$ \\
\hline $\begin{array}{ll}\text { Less than } & \frac{1}{2} \\
\frac{1}{2}- & \cdots \\
1- & \cdots \\
1 \frac{1}{2}- & \cdots \\
2- & \cdots \\
2 \frac{1}{2}- & \cdots \\
3- & \cdots \\
3 \frac{1}{2}- & \cdots \\
4 \frac{1}{4}- & \cdots \\
5 \frac{1}{2}- & \cdots\end{array}$ & $\begin{array}{l}\cdots \\
\cdots \\
\cdots \\
\cdots \\
\cdots \\
\cdots \\
\cdots \\
\cdots\end{array}$ & $\begin{array}{l}11 \\
25 \\
27 \\
36 \\
24 \\
18 \\
16 \\
18 \\
13 \\
11 \\
24\end{array}$ & $\begin{array}{r}5 \cdot 3 \\
19 \cdot 8 \\
29 \cdot 4 \\
33 \cdot 5 \\
29 \cdot 8 \\
26 \cdot 6 \\
21 \cdot 5 \\
16 \cdot 4 \\
10 \cdot 2 \\
8 \cdot 2 \\
21 \cdot 8\end{array}$ \\
\hline Totals & $\ldots$ & 223 & $222 \cdot 5$ \\
\hline
\end{tabular}

TABLE II

MEAN AND STANDARD DEVIATION OF TIMES FOR CHEST RADIOGRAPHY OF PATIENTS GROUPED BY AGE: SAMPLE OF ONE WEEK'S WORK

\begin{tabular}{|c|c|c|c|c|c|c|}
\hline \multirow[b]{2}{*}{ Age } & \multicolumn{3}{|c|}{$\begin{array}{c}\text { Patients on Ordinary } \\
\text { Machine }\end{array}$} & \multicolumn{3}{|c|}{ All Patients } \\
\hline & No. & $\underset{(\min )}{\text { Mean }}$ & $\underset{(\min )}{\text { SD }}$ & No. & $\underset{(\min )}{\text { Mean }}$ & $\underset{\text { (min) }}{S D}$ \\
\hline $\begin{array}{l}0- \\
10- \\
20- \\
30- \\
40- \\
50- \\
60- \\
70- \\
80- \\
90-\end{array}$ & $\begin{array}{r}1 \\
10 \\
7 \\
15 \\
37 \\
36 \\
57 \\
48 \\
11 \\
1\end{array}$ & $\begin{array}{l}3 \cdot 75 \\
2 \cdot 39 \\
2 \cdot 74 \\
2.48 \\
2 \cdot 26 \\
2 \cdot 28 \\
2 \cdot 69 \\
3.59 \\
4 \cdot 52 \\
4 \cdot 83\end{array}$ & $\begin{array}{r}1.58 \\
1.13 \\
1.02 \\
1.22 \\
1.28 \\
1.68 \\
2.07 \\
1.39 \\
-\end{array}$ & $\begin{array}{r}1 \\
21 \\
10 \\
25 \\
41 \\
38 \\
60 \\
48 \\
11 \\
1\end{array}$ & $\begin{array}{l}3 \cdot 75 \\
1 \cdot 73 \\
2 \cdot 15 \\
2 \cdot 21 \\
2 \cdot 19 \\
2 \cdot 27 \\
2 \cdot 64 \\
3 \cdot 59 \\
4 \cdot 52 \\
4 \cdot 83\end{array}$ & $\begin{array}{r}1 . \overline{49} \\
1.00 \\
0.94 \\
1.18 \\
1.28 \\
1.68 \\
2.07 \\
1.39 \\
\end{array}$ \\
\hline
\end{tabular}

the variance for patients in the age range 20-59 years. This is probably because these groups contain comparatively high proportions of cases where the patient was slower to respond to direction from the staff during the examination (this was very noticeable in children and senile patients) or where other difficulties, such as limited patient mobility, were encountered. When the data are grouped by patient origin and mobility, as in Table III, some other tendencies are evident. The service mean and variance both increase substantially with decreasing mobility and fitness of the patient; for example, trolley patients take almost twice as long on average as walking patients and are also far more variable. The miniature machine gave shorter examination

TABLE III

MEAN AND STANDARD DEVIATION TIMES FOR CHEST RADIOGRAPH OF VARIOUS PATIENT GROUPS: SAMPLE RADIOGRAPH OF OF ONE WEEK'S WORK

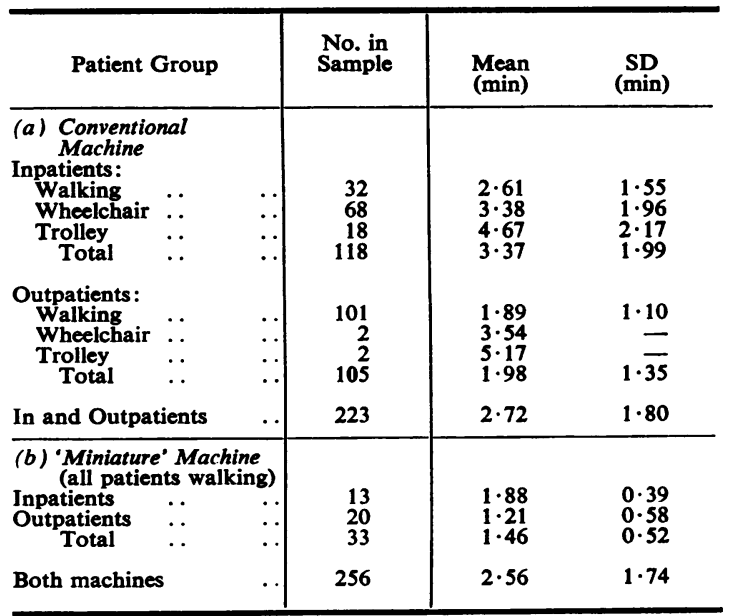


completion times, but there were no significant differences in the means or variances of comparable patient groups of opposite sex.

It is well recognized that in a queueing system, to increase efficiency it is desirable to deal with incoming units which have a homogeneous service time distribution; if there are some units with markedly different characteristics from the rest, it is preferable to deal with them separately to allow for these differences. In particular, in the hospital clinic, it is preferable to deal separately with patients who have a different mean examination time; if this is done, an appointment schedule may be adopted having different appointment intervals for the various groups of patients. We are thus driven to consider ways of segregating patients into less heterogeneous groups. It is difficult to envisage an operating policy which completely segregated patients according to the mobility and age factors, as these details often are not known before the patient arrives at the clinic. However, this form of segregation is used when dealing with exceptionally old and difficult patients towards the end of a session, thus causing less congestion to any subsequent patients.

Segregation by origin of the patient is feasible, and in fact is used in practice to some extent already when $x$-ray facilities are set aside for the use of patients from a particular source at certain times (a room reserved during selected working hours of a fracture clinic, for example). When the distributions

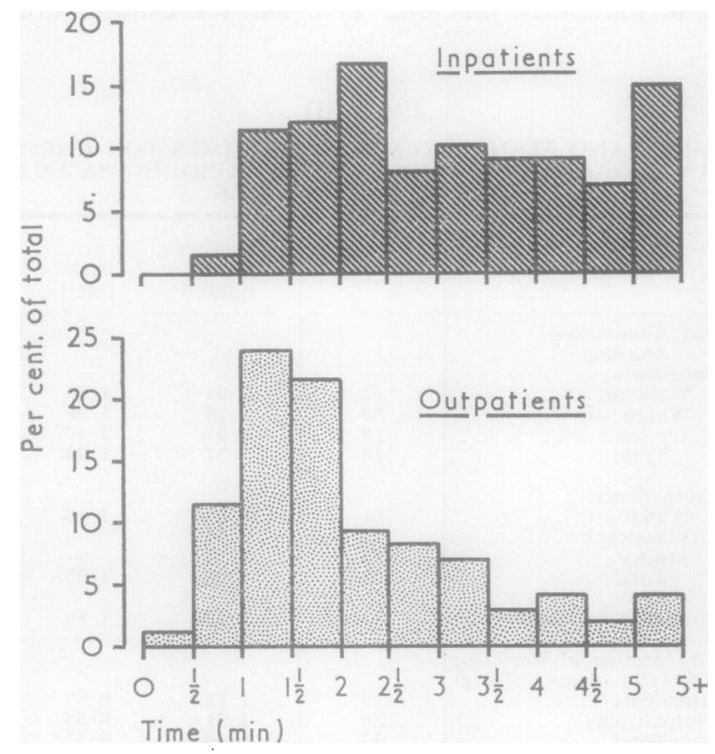

FIG. 1. Sample distribution of times to complete chest $x$-ray examinations of examination times are formed for inpatients and outpatients, the differences in age distribution and mobility levels produce the data in Fig. 1. We may see that inpatients have a substantial proportion of 'long' examinations (over five minutes), and that the inpatients have a more skewed pattern with a relatively small number of intermediate and long examination times. These obvious differences warrant an investigation of the consequences of segregating patients in this way. It would not be possible in practice to time-table work entirely in sessions dealing with patients from a small number of specified sources, but the general idea may be worth pursuing, even though the patient's origin is not a cause of variation in the service time, as are his age and mobility. However, we first consider the simulation description of a department dealing with a mixed input of patients. Although space does not allow a lengthy description of the model here, the simulation program listing and specification is available on request. The important parameters to be varied are:

$N=$ clinic size, or the total number of patients examined during one session

$I=$ number of patients with appointments called at the start of each session

$r=$ rate of arrival of patients with appointments rate of arrival of patients without appointments

$m=$ appointment batch size, or the number of patients called at each appointment time

$k=$ index of variability of the gamma type service time distribution (variability decreases with increasing $k$ )

The duration of the clinic was taken in all cases to be two and a half hours. Thus a chest $x$-ray clinic, with approximately the same service time mean as quoted in Table III, would have a size slightly less than 50 patients when idle time and other minor sources of delay are taken into account. There are indications that other more complex examinations have service time distributions which are approximately gamma (Fraser, 1969) and, although no detailed data are available, it would be reasonable to expect some sort of unimodal, positively skewed distribution; this being so we may include in the simulations clinics of smaller size and larger mean service time, and expect the results to be approximately true. The patients are served in a 'first-come, first-served' discipline, and it is assumed that they arrive, on average, at the same rate as that at which they can be examined. 


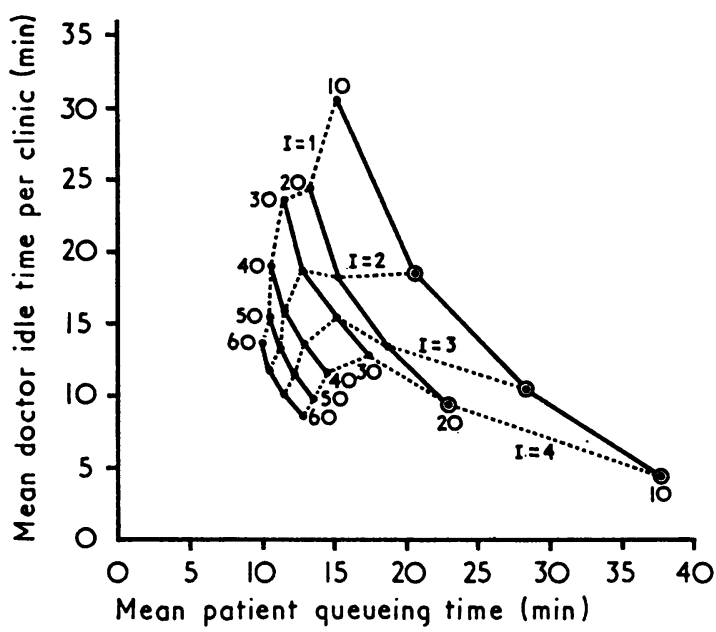

Fig. 2. Results of simulations for $r=1 \cdot 0, k=2, m=1$. Small numbers refer to clinic size $\mathbf{N}$.

\section{RESULTS}

Considering simulations for a typical set of parameter values, the results may be shown as in Fig. 2, using the doctor (or staff) mean idle time per clinic and the mean patient queueing time as measures of efficiency; the lines joining the plotted points are for clarity of presentation only. Each plotted point gives the average statistics from 25 simulated clinic sessions; this was found to give coefficients of variation for the means not greater than $5 \%$. We may note the following points, some of which are to be expected intuitively: higher values of $I$ (the initial number of patients) always give smaller idle time and higher queueing time in clinics of the same size $N$; in general, clinics with the same value of $I$ show idle and queueing times both decreasing as $N$ increases, but there are areas in the higher values of $I$ where size increases are associated with a slightly increasing idle time; the effect of $I$ is smaller in large clinics than in small ones. The circled points on the figure represent clinics which did not meet the Ministry of Health's suggested standard, that 'not more than $25 \%$ of patients should wait more than $\mathbf{3 0}$ minutes'. Figures corresponding to clinics having other characteristics may be plotted in the same way. As the parameters are varied, the cross-section of the multivariate response surface shown in Fig. 2 shows the same basic shape but distorts as the effects of particular changes in the parameter values are felt. For example, if $k$ is reduced (corresponding to having a more variable service time distribution), increases in both idle time and queueing time may be seen in comparable clinics, and as $k$ increases, the patient mean queueing time becomes almost constant, the idle time reducing with increasing clinic size. Fig. 3 shows what happens if $r$ is reduced to 0.5 (that is there are now twice as many non-appointment patients as appointment patients). The points corresponding to each clinic in Fig. 2 have moved 'north-east', implying an increase in both idle time and queueing time. In contrast, a higher value of $r$ may be shown to give more efficient schedules from both staff and patient points of view. These and further results show that, for low values of $I$, it is idle time that is more affected by $N$, whereas, with higher values, it becomes the patient queueing time which shows the greater change. (As the clinic length is constant, different clinic sizes also imply a different mean examination time; the comparison of clinics with different sizes, but the same mean examination time, is discussed later.)

Fig. 4 shows the effect of altering $r$ while the batch size $m$ and the initial number $I$ are kept constant. The meaningful comparisons here are between clinics of the same size, and it may be seen in almost all cases that an increase in $r$ results in an increase in either patient queueing time or doctor idle time, or both. We may clearly see here that when the proportion of appointment patients increases, in small clinics it is the doctor who benefits by a reduction in his expected idle time, and for larger clinics it is to the patient's advantage because there is a decrease in their expected queueing time. These results agree with the findings of a

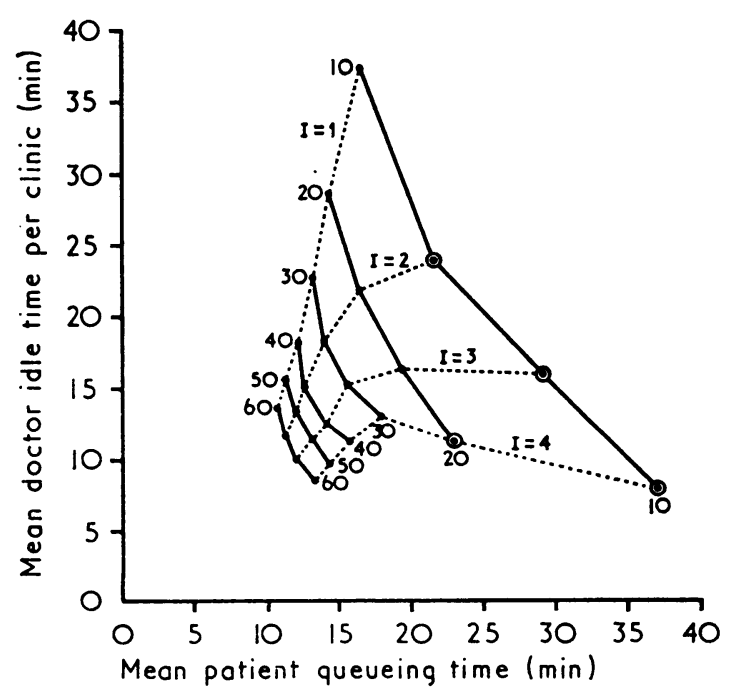

Fig. 3. Results of simulations for $r=0.5, k=2, m=1$. Small numbers refer to clinic size $\mathbf{N}$. 


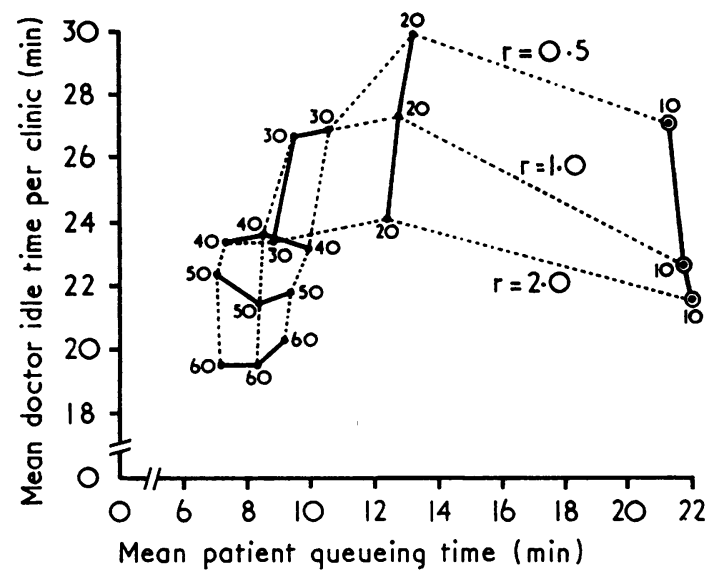

Fig. 4. Results of simulations for $k=1, m=1, I=2$. Small numbers refer to clinic size $\mathbf{N}$.

similar theoretical model where, assuming the system is in statistical equilibrium, it can be shown that increases in $r$ are always associated with decreases in the expected queueing time for all patients (Walter, 1972).

Fig. 5 demonstrates the effect of the appointment batch size $m$, or the number of patients allotted to

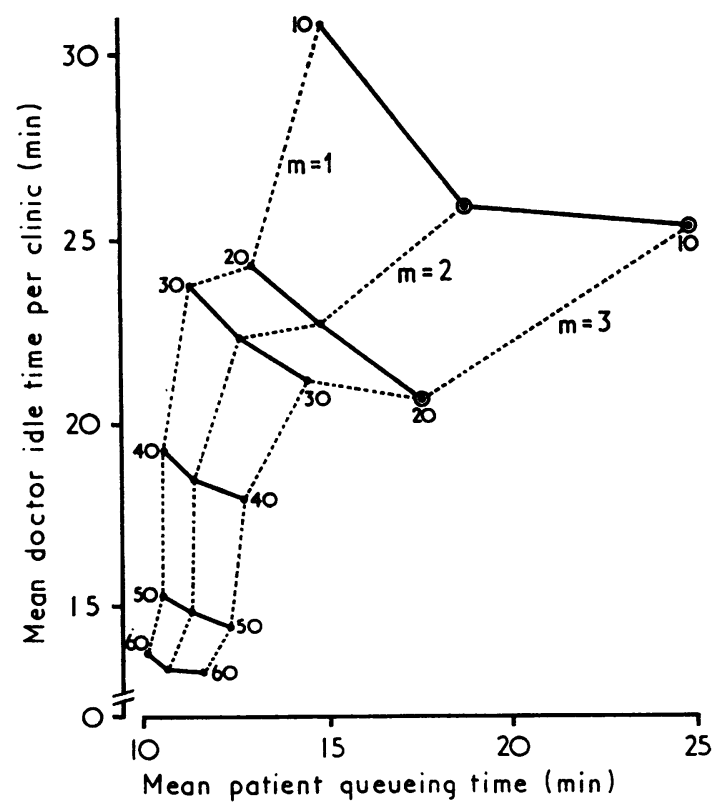

Fig. 5. Results of simulations for $\mathrm{r}=1 \cdot 0, \mathrm{k}=2, \mathrm{~m}=1$. Small numbers refer to clinic size $\mathbf{N}$. each appointment time. For a valid comparison between appointment schedules, it is inadmissible to keep the initial number $I$ constant, as this number represents a particular block of appointment patients who happen to be scheduled for the start of the session. In practice, any increase in $m$ would be accompanied by an increase in $I$; therefore, $I$ was taken as being equal to $m$ for this comparison only. We may see that in large clinics, the effect of $m$ is small; if anything, a larger value of $m$ seems to be against the patients' interest (as queueing time is slightly increased and idle time is slightly decreased). When we consider medium-sized clinics, the differences become more pronounced; as an example, $\mathbf{N}=30$ shows a 'south-easterly' movement (decreasing idle time and increasing queueing time) with increasing $\boldsymbol{m}$. In general, successive increases in $\boldsymbol{m}$ yield progressively smaller reductions in the idle time and larger increases in queueing time (a diminishing return effect for the doctor), although there are exceptions to this. Therefore, although batch sizes other than one may be justifiable because the idle time is reduced, we must remember that an increase in queueing time affects each patient in the session; also $m$ must not be so large that only small reductions in idle time are bought at the expense of large increases in queueing time. This is particularly true for small clinics where the queueing time may increase substantially even between $m=1$ and $m=2$; in this situation it is very hard (on this basis) to justify a choice of batch size other than one. However, clinics with a highly variable service time distribution do seem to profit from batch bookings, and there may be considerable practical merit in giving "rounded" appointment times, for example calling two patients at each quarter-hour, rather than one patient every $7 \frac{1}{2}$ minutes.

If it were possible to establish a numerical ratio for the relative values of the times wasted by the doctor and an 'average' patient, one could use results of this kind to pick parameter values giving an optimal appointment schedule in terms of balancing the two. Fig. 6 shows the results for $r=1, k=4, m=1$, with the abscissa taken now as the total queueing time by all patients in the session. If we stated that the doctor's time was worth $\lambda$ times that of one of his patients, we would wish to choose the appointment schedule which minimized the value of the total time wasted by the doctor and his patients, that is, $x+\lambda y$, where $y$ is the total idle time and $x$ is the total patient queueing time, for a given size of clinic. As an example, if $\lambda$ is taken as 10 , the best systems are obtained when $I=3$ for $N=10$ or 20 , and $I=2$ for $N=30$ or more. A smaller value of $\lambda$ gives smaller values of $I$, 


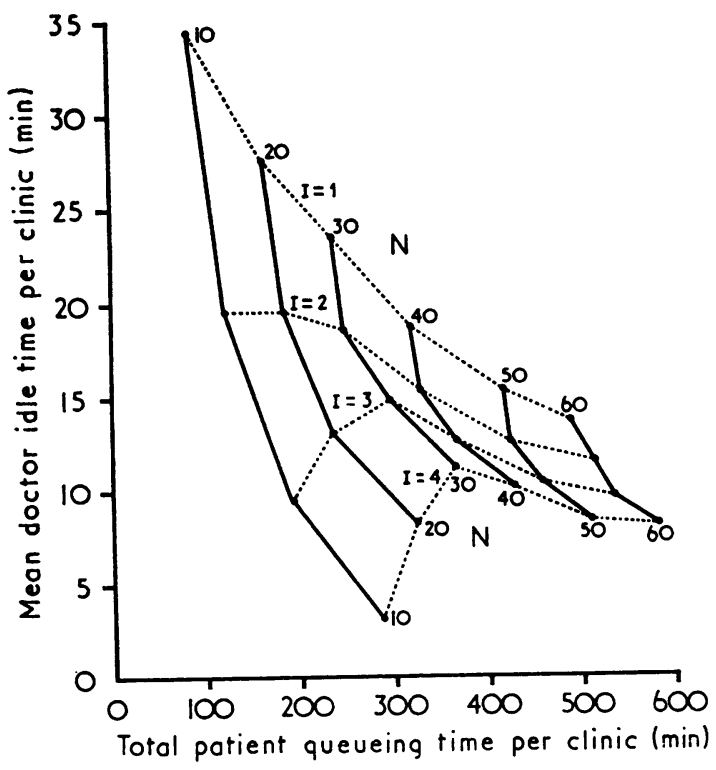

FIG. 6. Results of simulations for $r=1 \cdot 0, k=4, m=1$. Small numbers refer to clinic size $\mathrm{N}$.

that is, the initial patient batch is reduced in size, and consequently the subsequent queueing times are reduced.

As mentioned previously, the mean examination time is taken always as $150 / N$ minutes; however, we may also consider the mean idle time and the total patient queueing time for clinics with the same mean examination time, $\mu$, say, by scaling the results of Fig. 6 by the factor $\mu N / 150$. Alternatively, we may again use the mean queueing time per patient by scaling by $\mu / 150$; the idle time correspondingly becomes the idle time per patient seen. As an example, for a mean examination time of five minutes, and for $N=30, I=3$, we would estimate from Fig. 6 a mean queueing time per patient of approximately $300 \times 5 / 150=10$ minutes, and an idle time per patient of $15 \times 5 / 150=0.5$ minutes.

The results thus far have not related the service time distribution of a patient to his origin or other characteristics, as was suggested earlier; in other words, the same distribution has been used for all patients. Some further work was carried out dividing the patients into sessions consisting mainly of either inpatients or outpatients. As a development of the above work, it was assumed, somewhat arbitrarily, that $95 \%$ of inpatients and $5 \%$ of outpatients have appointments, although other proportions could easily be incorporated in this methodology for a given hospital. It is worth repeating that total segregation is difficult to envisage in practice; at best one could hope to see work sessions consisting of segments of work of mostly inpatients or mostly outpatients. To relate the examination time to the patient's origin, as the patient 'arrived' in the simulation program execution, his origin was generated using the known composition of the incoming work; then a service time was selected from the appropriate distribution of Fig. 1. The general appearance of the idle timequeueing time response surface was much the same as in simulations with an age-independent examination time distribution. The patient queueing times were roughly the same in the inpatient and outpatient sessions, but the idle time in the inpatient session was approximately half that in the corresponding outpatient session.

Further clinics were simulated having sessions which consisted of two equal segments, the first having a patient composition as in the 'inpatient' session above, and the second as in the 'outpatient' session. It was found that when these were compared with clinics where no segregation was used, the total queueing time by patients was almost the same; however, the total idle time was reduced by as much as $30 \%$. Although even simulation models are only crude representations of reality, a saving of the same order as this in the use of the time of highly trained medical personnel would result in immense financial economies in the health service; further, these improvements may be made by a relatively simple rearrangement of the work schedule.

\section{Discussion}

Hospital clinic appointment schemes exist in order to make efficient use of the working time of trained medical personnel, while at the same time reducing the levels of inconvenience for the patients. The value of an appointment scheme might be assessed directly by controlled experimentation, but this may be difficult or impossible in practical terms. An alternative approach is to use a simulation model to predict the effect of possible innovations in working procedures.

The rapid growth in the size and power of the electronic computer has been accompanied by an increasing interest in its uses in medicine and its administration. It is now possible to build simulation models to describe organizational structures as complicated as a hospital, and these may be used to monitor the efficiency indices of the system. A further use has been demonstrated here in the 
simulation of a simplified system to provide general policy guidelines in the clinic situation. Stress should not be laid on the accuracy of the numerical results because these would be substantially different in any real situation with a large number of minor factors which are difficult to model accurately; however, if the basic structure of the model is realistic, then comparisons of different administrative regimes to be used in the real world are justifiable.

Considering the results from simulated systems treating all types of patient in a given clinic session, it was found that efficiency always improves when the proportion of patients with appointments is increased, through a reduction in either the doctor's idle time or the patient's queueing time, or both. In small clinics it is the doctor who benefits most from such a step, and in large clinics it is the patients who gain. The behaviour of the queueing system throughout the duration of the session depends greatly on the number of patients present at the start, particularly in small clinics. It also appears that the practice of giving multiple bookings for a given appointment time yields a small benefit to the staff by a reduction in idle time, but at the cost of substantially increasing the mean queueing time. It can be argued that multiple bookings guard against patients failing to arrive at their appointment time; however, it has been shown (Blanco White and Pike, 1964) that when there is a proportion of defaulters which is not too large, the efficiency of the clinic can be maintained simply by making a suitable adjustment to the average time between appointments. The choice of parameter values to define an efficient appointment schedule in small clinics is a difficult one, and it may be necessary to resort to a simple cost-benefit analysis to obtain a rational solution to the problem; it is still true, however, that increases in the proportion of patients seen by appointment are desirable.

Turning to the possibility of segregating patients into different sessions in order to allow for the differences in their examination time distributions, it was found that substantial savings in staff time may be made even by a simple grouping into inpatients and outpatients. The methods used here could be refined to describe any hospital radiology department with a known patient input, and to predict the effects of modifications to the daily routine. All departments keep accurate records of the examinations performed, and so it would be a simple matter to determine the parameters defining the constitution of the patient input. Some hospitals also keep routine records of the time each patient spends in the department; using data from this source, or from a small works study, it should be possible to determine the approximate examination time distributions for the various patient groups, and thus derive an appointment scheme which is both efficient and practical.

\section{SUMMARY}

Relative to other stochastic systems seen in hospitals, the patient queueing process of a radiology department is difficult to describe theoretically because of its wide variety of work sources, and in particular because of the mixing of patients who arrive with and without appointments. Using empirical data on $x$-ray completion times, a computer simulation model was built and used to predict the effects of administrative changes on efficiency, as measured by the average patient queueing time and doctor idle-time during a clinic session. Efficiency always improves when the proportion of patients with appointments is increased, but whether it is the staff or patients who benefit depends on the clinic size; the behaviour of the system depends critically on the number of patients called at the beginning of the session, particularly in smallero clinics. In most circumstances, patients should be given coincident appointment times only for reasons of practicality. The completion time of a patient is shown to depend strongly on his age, mobility, and origin; this is exploited to demonstrate the efficiency increases to be expected when clinic sessions consist of groups of patients from the same origins.

I would like to thank Professor D. J. Finney, F.R.S. of the Department of Statistics, University of Edinburgh, and Professor Eric Samuel, Department of Diagnostic Radiology, Royal Infirmary of Edinburgh, for their help and encouragement in the execution of this work, and the referees for their helpful comments on an earlier draft of this paper.

\section{REFERENCES}

BaILEY, N. T. J. (1952). A study of queues and appointment systems in hospital out-patient departments, with special reference to waiting-times. J. roy. Statist. Soc., (B), 14, 185.

Blanco White, M. J., and Pike, M. C. (1964). Appointment systems in out-patients' clinics and the effect of patients' unpunctuality. Med. Care (Lond.), 2, 133. 
FrASER, B. J. (1969). The organisation of a radiology department in a district general hospital. Ph.D. thesis, University of Reading.

Jeans, W. D., Berger, S. R., and Gill, R. (1972). Computer simulation model of an $x$-ray department. Brit. med. J., 1, 675.

Nuffield Provincial Hospitals Trust (1962). Towards a Clearer View; the Organization of Diagnostic X-Ray Departments. Oxford University Press, London.
PIKe, M. C. (1963a). Some numerical results for the queueing system $\mathrm{D} / \mathrm{E}_{\mathrm{k}} / 1$. J. roy. Statist. Soc. (B), $25,477$.

(1963b). Waiting in hospital outpatient and casualty departments. Ph.D. thesis, University of Aberdeen.

WALTER, S. D. (1972). A statistical investigation of procedure in hospital clinics. Ph.D. thesis, University of Edinburgh. 